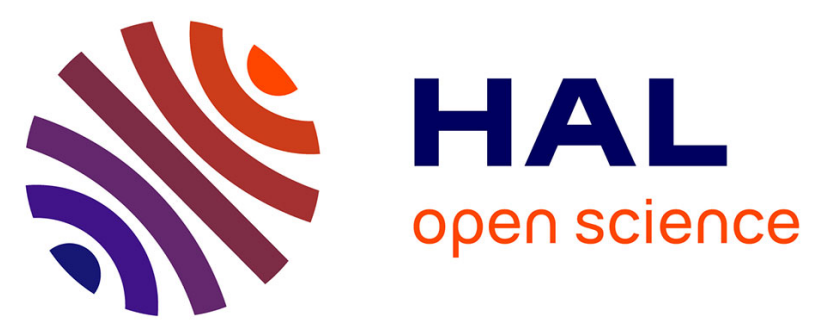

\title{
Haplotype selection as an adaptive mechanism in the protozoan pathogen Leishmania donovani
}

Pablo Prieto Barja, Pascale Pescher, Giovanni Bussotti, Franck Dumetz, Hideo Imamura, Darek Kedra, Malgorzata Domagalska, Victor Chaumeau, Heinz Himmelbauer, Michel Pagès, et al.

\section{To cite this version:}

Pablo Prieto Barja, Pascale Pescher, Giovanni Bussotti, Franck Dumetz, Hideo Imamura, et al.. Haplotype selection as an adaptive mechanism in the protozoan pathogen Leishmania donovani. Nature Ecology \& Evolution, 2017, 1 (12), pp.1961-1969. 10.1038/s41559-017-0361-x . pasteur-02107201

\section{HAL Id: pasteur-02107201}

https://hal-pasteur.archives-ouvertes.fr/pasteur-02107201

Submitted on 23 Apr 2019

HAL is a multi-disciplinary open access archive for the deposit and dissemination of scientific research documents, whether they are published or not. The documents may come from teaching and research institutions in France or abroad, or from public or private research centers.
L'archive ouverte pluridisciplinaire HAL, est destinée au dépôt et à la diffusion de documents scientifiques de niveau recherche, publiés ou non, émanant des établissements d'enseignement et de recherche français ou étrangers, des laboratoires publics ou privés.

\section{(2)(1) $\$$}

Distributed under a Creative Commons Attribution - NonCommercial - ShareAlikel 4.0 
3 Pablo Prieto Barja ${ }^{1,6, \$}$, Pascale Pescher ${ }^{2, \$}$, Giovanni Bussotti ${ }^{3}$, Franck Dumetz ${ }^{4}$, Hideo

4 Imamura ${ }^{4}$, Darek Kedra ${ }^{1}$, Malgorzata Domagalska ${ }^{4}$, Victor Chaumeau ${ }^{5}$, Heinz

5 Himmelbauer ${ }^{1,7}$, Michel Pages ${ }^{5}$, Yvon Sterkers ${ }^{5}$, Jean-Claude Dujardin ${ }^{4,8}$, Cedric

6 Notredame ${ }^{1,6,{ }^{*}} \&$ Gerald Frank Späth ${ }^{2,{ }^{*}}$

7

$8{ }^{1}$ Centre for Genomic Regulation (CRG), The Barcelona Institute of Science and Technology,

9 Barcelona, Spain; ${ }^{2}$ Institut Pasteur, INSERM U1201, Unité de Parasitologie moléculaire et

10 Signalisation, Paris, France; ${ }^{3}$ HUB de Bioinformatique et Biostatistiques, Centre de

11 Bioinformatique, Biostatistique et Biologie Intégrative (C3BI), Institut Pasteur, Paris, France;

$12{ }^{4}$ Institute of Tropical Medicine, Molecular Parasitology Unit, Antwerpen, Belgium; ${ }^{5}$ University

13 Montpellier, Faculty of Medicine, Laboratory of Parasitology-Mycology, CNRS 5290, IRD

14 224, University Montpellier (UMR “MiVEGEC”) and Centre Hospitalier Universitaire,

15 Montpellier, France; ${ }^{6}$ Universitat Pompeu Fabra (UPF), Barcelona, Spain; ${ }^{7}$ Institute of

16 Biotechnology Muthgasse 181190 Vienna; ${ }^{8}$ Universiteit Antwerpen, Department of

17 Biomedical Sciences, Belgium

18

19 Running title: L. donovani haplotype selection

20

$21{ }^{*}$ Corresponding authors:

22 G. F. Späth, Tel: 01.40.61.38.58; E-mail: gerald.spaeth@pasteur.fr

23 C. Notredame, Tel: +34 933160271; Email: cedric.notredame@crg.eu

24 these authors contributed equally to this work 
26 Keywords: Leishmania donovani, aneuploidy, genome instability, dosage compensation,

27 fitness, haplotypes

\section{Summary}

32 The parasite Leishmania donovani causes a fatal disease termed visceral leishmaniasis.

33 The process through which the parasite adapts to environmental change remains largely

34 unknown. Here we show that aneuploidy is integral for parasite adaptation and that

35 karyotypic fluctuations allow for selection of beneficial haplotypes, which impact

36 transcriptomic output and correlate with phenotypic variations in proliferation and infectivity.

37 To avoid loss of diversity following karyotype and haplotype selection, L. donovani utilizes

38 two mechanisms: polyclonal selection of beneficial haplotypes to create coexisting

39 subpopulations that preserve the original diversity, and generation of new diversity as

40 aneuploidy-prone chromosomes tolerate higher mutation rates. Our results reveal high

41 aneuploidy turnover and haplotype selection as a unique evolutionary adaptation mechanism

42 that $L$. donovani uses to preserve genetic diversity under strong selection. This unexplored

43 process may function in other human diseases, including fungal infection and cancer, and

44 stimulate innovative treatment options. 
51 Rapid pathogen adaptation to novel environments is a major threat to human health. In

52 parasites evolving under constant host selection, fitness gains often cause increased

53 pathogenicity ${ }^{1}$. Aneuploidy recently emerged as a key driver for evolutionary adaptation of

54 fungal and protist pathogens ${ }^{2-4}$, in which chromosome copy number variations induce

55 phenotypic changes through modulation of transcript and protein abundance ${ }^{5}$. In addition to

56 its effect on gene count, aneuploidy impacts cellular phenotypes through the selection of

57 beneficial alleles. While this phenomenon has received considerable attention in human

58 genetics $^{6}$ and cancer genome analysis ${ }^{7,8,9}$, the role of aneuploidy on allelic selection in

59 genome evolution and adaptation in pathogenic eukaryotes remains poorly understood in

60 most organisms, in spite of recent attention ${ }^{10,11}$.

61 We addressed this question in the protozoan parasite Leishmania donovani, an

62 important human pathogen that causes fatal visceral leishmaniasis ${ }^{12}$. During its life cycle

63 Leishmania undergoes a major developmental transition from insect-stage promastigotes to

64 mammalian-stage amastigotes, which adapts these parasites for extra- and intracellular

65 survival, respectively ${ }^{13}$. In addition to environmentally-induced stage differentiation,

66 Leishmania can adapt to a variety of unpredictable fluctuations inside its human host,

67 notably pharmacological interventions. Such environment-genotype interactions likely select

68 parasites for higher fitness and have important consequences on disease outcome as

69 demonstrated by the emergence of drug resistant clinical isolates ${ }^{14,15}$. However, the

70 underlying genetic mechanisms that drive short-term Leishmania evolution are still largely

71 unknown. In the absence of classical transcriptional gene regulation, these early-branching

72 eukaryotes often control protein abundance via gene and chromosome amplification ${ }^{10,16}$.

73 This process is fueled by frequent asymmetric chromosome allotments, which cause 
74 constitutive mosaicism ${ }^{17,18}$. This model has recently received new support ${ }^{19}$ with a large-

75 scale deep sequencing study of $L$. donovani field isolates showing a correlation between

76 polysomy variations and changes in heterozygosity. Leishmania therefore represents an

77 ideal non-conventional system to investigate how karyotype variations and allelic selection

78 drive parasite fitness gains and how transient and stable aneuploidies impact the long-term

79 evolutionary trajectory.

80

\section{$81 \quad$ Results}

\section{Karyotype and haplotype selection in field isolates}

83 We first explored the impact of aneuploidy on allelic selection in Leishmania using the

84 recently published sequencing data of 204 L. donovani field isolates ${ }^{19}$. These populations

85 originate from independent evolutionary radiations that occurred after a population

86 bottleneck caused by DDT vector control campaigns in the 1960s. This makes them a

87 convenient benchmark to study genetic diversity dynamics. We conducted read-depth

88 analyses on publicly available sequencing data $\operatorname{sets}^{19}$ to explore ploidy variations across

89 culture-adapted isolates (Fig. 1a). Even though a large number of intermediate read-depth

90 values suggest frequent mosaicism (Fig. 1a, Supplementary Table 1), the relatively high

91 frequency of discrete polysomy levels other than disomic levels (notably trisomy) indicates

92 that a large fraction of isolates may be homogenous for single or co-occurring aneuploidies

93 (e.g chr 5, 9, 23 and 26, Fig. 1b). While all chromosomes are predominantly disomic except

94 for chr 31 , the overall karyotypic diversity is very high with the most frequent karyotype

95 representing only about $10 \%$ of the 204 isolates.

96 The frequent occurrence of karyotypic similarities among isolates suggests that the 
97 corresponding aneuploidies may either have a common, monoclonal origin (i.e. one unique

98 founding duplication) or result from frequent convergent processes (i.e. selection of several

99 independent duplications of the same homologous chromosome). Relatively weak

100 correlations between aneuploidy and SNP variation ${ }^{19}$ does not discriminate between these

101 two possibilities. While clonal expansion of a single founder may underlie the most common

102 aneuploidies (like chr 31,5 or 26), their occurrence in karyotypically very diverse isolates is

103 also compatible with a polyclonal origin.

104 Under a polyclonal scenario, each culture-adapted isolate arises from the expansion

105 of mixed subpopulations sharing some independently generated polysomies. This model is

106 in good agreement with mosaicism, previously observed in cultured Leishmania major ${ }^{18}$. We

107 analyzed allelic frequency distributions to evaluate the likelihood of polyclonal origin

108 (Supplementary Fig. 1). In this analysis, well-defined peaks in the merged profiles of most

109 chromosome allelic frequency distributions indicate that most heterozygote alleles are

110 balanced similarly across isolates. For disomic profiles, we typically observed unimodal

111 allelic frequency distributions centered at 50\% (Supplementary Fig. 1a - left column). These

112 observations are consistent with diploid asexual populations in which most alleles diffuse by

113 random drift.

114 Chromosomes with a read-depth level compatible with trisomy present us with a

115 more complex situation. In these chromosomes, the merged allelic frequency profiles

116 (Supplementary Fig. 1a - right column) revealed a continuum between the two extreme

117 cases of pure unimodal (e.g. chr 1, Fig. 1c, Supplementary Fig. 1d bottom scenario) and

118 pure bimodal (e.g. chr 5, Fig. 1c, Supplementary Fig. 1d other scenarios) allelic frequency

119 distributions. In the absence of mosaicism, trisomies usually cause bimodal allelic frequency 
distributions. Unimodal distributions like chr 1 therefore reflect population mosaicism as a

121 consequence of a duplication scenario with both homologous chromosomes equally likely to

122 be duplicated across the entire population of cells constituting an isolate, thus maintaining

123 the original allelic frequency equilibrium of the disomic state (Supplementary Fig 1d). The

124 coexistence of haplotypically different trisomies within the same isolate clearly indicates

125 mosaicism, which strongly suggests a polyclonal origin. In contrast, bimodal allelic frequency

126 distributions centered on 33 and $66 \%$, like that observed for chr 5 , reflect the collective

127 doubling in relative frequency of half of the alleles and are characteristic of homogenous

128 duplication of the same homologous chromosome within each isolate population (thus

129 defining an identical haplotype). While the integration of various mosaicism combinations by

130 sequencing analysis could cause similar read-depths, it is unlikely that mosaicism would also

131 cause bimodal allelic frequency distributions centered on 33 and $66 \%$.

132 Even though they occur in a large fraction of the isolates, chr 5 trisomies are most

133 likely the result of a convergent process as suggested by their polyphyletic dispersion on the

134 phylogenetic tree of the 204 filed isolates ${ }^{19}$. Given such a convergent process, we asked if

135 these trisomies were selected as karyotypes or as specific haplotype combinations.

136 Haplotype selection should cause increased homogeneity across isolates. We therefore

137 measured allelic dispersion across trisomic isolates. We estimated the relative loss of

138 heterozygosity ( $\mathrm{LOH}$ ) between disomic and trisomic isolates for each chromosome. This

139 analysis shows that chr 5 had one of the lowest allelic diversity (highest average LOH) and

140 suggests frequent selection of the same homologous chromosome among the trisomic

141 isolates.

142 On these same subsets of trisomic and disomic chromosomes, we measured the 143 segregation variation (SV). SV is defined as the average ratio between the frequency of the 
144 dominant allele in each trisomic isolate and its corresponding average frequency in disomic

145 isolates. This index reflects the changes in segregation patterns between disomic and

146 trisomic states and is highest for chromosomes that show a unimodal distribution at a

147 disomic state and a bi-modal distribution at a trisomic state. As expected, we found chr 5 to

148 have one of the highest SV ratio. We also found a strong correlation between LOH and SV

149 across all chromosomes with the scatter plot displaying a clear continuum between chr 1

150 and chr 29 (Fig. 1d). This observation is in agreement with our hypothesis that high levels of

151 haplotype selection cause increased allelic homogeneity across isolates. These results

152 confirm that bimodal allelic frequency profiles are more likely induced by a selection process

153 rather than a monoclonal founding event. Monoclonal origin without haplotype selection

154 would cause a high SV and low LOH. Interestingly, distinctly bimodal chr 5 trisomies coexist

155 with unimodal trisomies of other chromosomes in a subset of the isolates (e.g. chr 12,

156 Supplementary Fig. 2). This finding indicates that various levels of haplotypic selection can

157 affect different chromosomes within the same parasite isolate. Altogether, the complex

158 karyotypic and haplotypic variations across field isolates clearly favors a polyclonal mosaic

159 origin as the most common scenario with diversity drawn from an equally diverse original

160 population, as postulated by Sterkers et al. ${ }^{18}$.

161

162 In vivo karyotype fluctuations

163 Polyclonal origin implies a pre-existing population-wide karyotypic mosaicism within each

164 individual isolate. Maintaining this degree of variability would require frequent polysomic

165 fluctuations. We measured this effect by monitoring chromosomal copy number when re-

166 passaging an aneuploid field isolate (strain LdBPK282) through hamsters. The rapid shift to

167 a predominant disomic karyotype in the field isolate analysed here (and an independent 
168 isolate shown by Dumetz et al. ${ }^{20}$ ) demonstrates highly dynamic aneuploidy fluctuations (Fig.

169 2a). It is unclear, however, if the observed aneuploidies during culture adaptation arise from 170 expansion of existing subpopulations or constitute a reversible de novo phenomenon. We

171 therefore examined this question applying single cell DNA-FISH analysis on liver and spleen

172 amastigotes. We analyzed the experimental Sudanese L. donovani strain LD1S with

173 available DNA-FISH probes to monitor aneuploidies for chr 5, 17, 22 and 27. Hamsters were

174 infected with amastigotes isolated from infected hamster spleens that showed a disomic read

175 depth for all chromosomes except tetrasomic chr 31 (see Supplementary Fig. 3a). For all

176 chromosomes investigated, our analysis confirmed frequent in situ mosaic aneuploidies in

177 liver and spleen (Fig. 2b). This pre-existing diversity can facilitate population-wide

178 aneuploidies in response to environmental changes. This possibility is in line with the mosaic

179 origin of aneuploidies observed in culture ${ }^{18}$.

180 In vitro karyotype and haplotype selection

181 While field isolate and in vivo analyses suggest that parasite adaptation relies on a

182 mechanism dedicated to accommodate frequent aneuploidies, these observations only

183 provide static snapshots of apparently highly dynamic fluctuations. To further elucidate this

184 process, we used again the experimental strain LD1S. In contrast to L. donovani clinical

185 isolates from the Indian sub-continent, this strain contains a larger number of heterozygous

186 sites (23014 compared to a median of 1143 per field isolate), which makes it an ideal model

187 for longitudinal monitoring of chromosome copy number variation and haplotype selection.

188 We used HTSeq to follow hamster-derived LD1S amastigotes during adaptation to in vitro

189 culture. Read depth analyses indicated that parasites rapidly establish stable trisomies for

190 chr 5, 9, 23, and 26 between in vitro passages p2 (ca. 20 generations) and p10 (ca. 60

191 generations) (Fig. 3a and Supplementary Fig. 3a). These karyotypic trajectories matched the 
most common variations observed in the field isolates (Fig. 1a and b, Supplementary Fig. 1) and they were highly reproducible across two independent experiments ${ }^{20}$. Not all aneuploidies are stable and homogenous, however. For example, chr 20 underwent a transient trisomy between passages p2 and p20 (ca. 190 generations), while probable mosaic aneuploidies (Fig. 3a) occurred for chr 14 and 15 and were stably maintained as judged by their intermediate read-depth levels at p10 and p20.

Population mosaicism in Leishmania major was previously examined on cultured promastigotes using a combination of sub-cloning and DNA-FISH analysis ${ }^{18}$. We used systematic comparison between p20 and eight individual sub-clones to model the original population complexity (Fig. 3b and Supplementary Fig. 3b). Haplotype and karyotype comparisons suggest that the eight clones may have arisen from at least two independent founding individuals (Supplementary Fig. 3c). Indeed, aneuploidies provide a powerful insight into haplotype phasing, due to systematic frequency shifts associated with duplicated homolog chromosome ${ }^{21}$. In this case, direct haplotype comparisons (Fig. 4a, Supplementary Fig. 4) clearly show how the allelic nature of chr 5 and chr 9 trisomies sets clones 1 and 8 apart from the rest. For instance, the existence of two groups with differences of major alleles on chr 5 ( 1 and 8 vs the rest) indicates that these trisomies were established through duplications of different chr 5 homologs (i.e. same chr 5 homologue duplicated in clones 1 and 8 that is different from the other clones). The most parsimonious reconstruction simply requires two trisomies of independent origin and is consistent with a high aneuploidy turnover $^{18}$. This explanation also applies to chromosome 9 and further supports the common origin of clones 1 and 8 . Sequencing 8 subclones has limited statistical power, and our speculation of two original founders does rely on a parsimonious hypothesis. Yet, the existence of clearly distinct subpopulations featuring similar aneuploidies further supports the 
216 mosaic origin scenario, under which individual strains represent complex mixtures of stable,

217 karyotypically distinct subpopulations.

218 Between subpopulations, similar karyotypes and haplotypes likely reflect a form of 219 convergence. At the karyotypic level, the most obvious indications of convergence occur on 220 chr 5, 9 and 26, whose trisomies are shared across all clones despite their independent 221 origins. Selection could also occur at the haplotypic level, with the clearest indication 222 provided by chr 26 whose haplotype map shows a consistent selection of the same allelic 223 combination across clones of different origin (Fig 4a). Unfortunately, the simultaneous 224 haplotype/karyotype selection taking place on this chromosome makes it impossible to 225 discriminate between purifying (lethality of one of the two possible trisomies) or positive 226 (higher fitness of one trisomy) selection pressure being imposed on its haplotype.

227 Chromosome 20 instead provides clear evidence for positive haplotype selection. This 228 chromosome is disomic and heterozygous at $\mathrm{p} 2$, becomes trisomic at $\mathrm{p} 10$, but reverts back 229 to disomy at p20 (Figs. 3a and 4b, Supplementary Fig. 5). After reversion, the allelic 230 frequency profiles unexpectedly show a near-perfect homozygote disomy in 6 out of 8 clones 231 (i.e. relatively flat allele frequency profile combined with a $60 \%$ decrease in the number of 232 heterozygous sites, Fig. 4a and Supplementary Fig. 6). In chr 20, transient trisomy therefore 233 provides an intermediate step to establish a homozygous disomy, whose selection 234 contributes to positive fitness as previously seen on yeast ${ }^{22}$.

\section{Correlation of aneuploidies with phenotypic variations}

237 Since Leishmania primarily lacks classical gene regulation mechanisms, it has long been speculated that polysomy should result in proportional transcriptional fluctuations ${ }^{15}$ and that aneuploidy mediated gene expression changes could be a trait under selection. We used 
240 HTSeq analysis to correlate read-depth levels between genome and transcriptome (Fig. 5a,

241 Supplementary Fig. 7) and found a very high correlation for most chromosomes $(r=0.72)$,

242 with the notable exception of chr 31. In this chromosome that is predominantly tetrasomic

243 across various Leishmania strains, transcriptomic output appears halved, suggesting some

244 form of dosage compensation, which cancels the polysomic effect. We also found karyotypic

245 fluctuations resulting from culture adaptation to correlate with rapidly increasing in vitro

246 fitness as judged by the decreasing generation time (Fig. 5b, left panel). Concurrently with

247 these variations the parasites showed decreased in vivo fitness as indicated by lower

248 infectivity (Fig. 5b middle and right panels). To assess if these variations take place during

249 the parasite life cycle, we separately sequence amastigotes obtained from spleen and liver

250 from one infected hamster. We found significantly distinct allelic profile variations for chr 20 ,

251 which showed a unimodal profile in liver and a bimodal profile in spleen (Fig. 5c,

252 Supplementary Fig. 8), while read depth analysis indicated a disomic state in both tissues

253 (Supplementary Fig. 9). These observations closely recapitulate our in vitro analyses of 254 parasite clones and indicate that transient polysomies may contribute to parasite adaptation 255 in vivo.

256

257 Long-term genetic implications on genetic diversity

258 Maintaining diversity under strong selection poses a dilemma for all microbial pathogens, 259 since it requires a mechanism that permits some compromise between immediate survival 260 through selection and long-term adaptation through the maintenance of genetic diversity. We 261 therefore searched for traces of increased genetic diversity associated with frequent 262 aneuploidies and found a clear signal in the 204 field isolates (Fig. 6). Integrating genetic 
variation across each individual isolate shows that polysomy-prone chromosomes exhibit a

264 significantly higher level of heterozygous sites than their more stable counterparts. This

265 trend is especially strong for chr 31 , which has the most stable, frequent and highest order

266 aneuploidy. This finding suggests that $L$. donovani might utilize aneuploidy to accumulate

267 mutations and increase its diversity. Further, these results confirm that even though transient

268 in vivo aneuploidies are difficult to detect and quantify, they are frequent enough to shape

269 the parasite genome and its genetic diversity.

270

271 Discussion

272 Utilizing recently published genomes of 204 field isolates ${ }^{19}$ and conducting evolutionary

273 experiments, we demonstrated that karyotypic variation modulates transcript abundance and

274 thus likely generates considerable phenotypic variability with possible fitness gains

275 associated with tissue-specific haplotype selection in situ. The genomic landscape defined

276 by an exhaustive combination of all possible trisomies, disomies and monosomies along with

277 their haplotype variations is substantial and at least one order of magnitude larger than a

278 similar estimate recently made on the basis of aneuploidy variations ${ }^{23}$. Aneuploidy turnover

279 therefore provides an efficient alternative to sex-based haplotype selection. This mechanism

280 may be especially useful within the mammalian host, where the absence of sexual

281 reproduction creates an even heavier selective pressure on the parasite's survival prospects.

282 Rapid aneuploidy turnover combined with haplotype selection permits fast adaptation

283 but comes with a significant genetic cost. Since alleles with lowered frequencies are at a

284 higher risk of disappearing from the genetic $\mathrm{pool}^{25}$, haplotype selection may induce loss of

285 heterozygosity. The transient trisomy of chr 20 and its in vivo, tissue-specific haplotypic

286 diversity illustrates the dilemma for the parasite between over-adaptation to any given 
287 environment that may involve irreversible genetic tradeoffs and maintenance of sufficient 288 genetic diversity for future adaptations. In this chromosome, the loss of heterozygosity could 289 contribute to adaptation across conditions and tissues, however this same process may also 290 yield an evolutionary dead end. The evolutionary success of Leishmania testifies of its ability 291 to establish a delicate balance between these conflicting requirements for short- and long292 term adaptation. Long-term survival for the parasite involves two complementary 293 mechanisms to generate and maintain genetic diversity. The first one relies on the mosaic 294 origin of selected populations. We demonstrated that culture adapted isolates do not have a 295 single founding parent but result from the simultaneous selection of several individuals. This 296 process maintains a high level of karyotypic and haplotypic diversity allowing polysomies to 297 occur frequently and independently in genetically distinct individuals. The second source of 298 diversity is a direct consequence of the relaxed selection that occurs after gene or 299 chromosome duplication ${ }^{26}$. Our findings agree with these models and further suggest that 300 aneuploidy-prone chromosomes have significantly higher mutation rates than their more 301 stable counterparts. Thus, aneuploidy represents an additional powerful mechanism to 302 increase genetic diversity in L. donovani.

$303 \quad$ Leishmania, like all known infectious agents, provides us with an excellent example 304 of genetic selection driven by survival. But it does so in a very unusual way: while high 305 mutation rates and genetic material exchange through sexual or analogous mechanisms 306 mediate survival in most pathogens, Leishmania evolved an additional strategy based on 307 genome instability to enhance parasite evolvability ${ }^{27-29}$. We show here that the parasite 308 harnessed this process and efficiently combined it with haplotype selection for rapid and 309 efficient genome adaptation under environmental pressure. This strategy has direct 310 consequences for current protocols in Leishmania drug and biomarker discovery. First, 
311 Leishmania genome instability limits all current and future drugs that directly target the

312 parasite biology and select for resistance phenotypes ${ }^{30,15,14}$. New strategies for anti-

313 leishmanial drug discovery should avoid direct parasite selection and rather target the

314 parasites' dependence on host cell metabolism. Second, the massive genomic changes we

315 observed during Leishmania culture adaptation challenges current protocols for biomarker

316 discovery, which all rely on in vitro expansion of clinical isolates. In the light of our findings,

317 we should modify these protocols to identify additional biomarkers hidden/diluted by culture-

318 based methods especially when polysomy itself is considered a biomarker. Alternatively, we

319 could establish culture-independent approaches that propagate field isolates in experimental

320 animal models or apply direct tissue sequencing. It is clear that genome instability needs to

321 be considered when investigating medically relevant aspects of the parasite's phenotype,

322 such as tissue tropism, drug susceptibility and pathogenicity. Our findings establish a

323 foundation for the future discovery of haplotypes with diagnostic and prognostic value.

324

325

326

327 
330 L. donovani strains, culture conditions and cell cloning. Culture-adapted L. donovani 331 field isolates maintained for more than 20 in vitro passages from the Indian Sub-Continent 332 were used in our analyses to track evolutionary diversity maintained across populations ${ }^{19}$. 333 Isolates were not sub-cloned and populations were used for all studies. Infectious $L$. 334 donovani strain 1S2D (MHOM/SD/62/1S-CL2D, referred to here as LD1S) was obtained 335 from Henry Murray (Weill Cornell Medical College, New York, USA) and maintained in hamsters by serial passaging (see below). All LD1S studies were performed with populations

337 except when specified otherwise. For promastigote differentiation and culture, $1 \times 10^{7}$ LD1S

338 amastigotes purified from hamster spleens were cultured at $26{ }^{\circ} \mathrm{C}$ in M199 media

339 supplemented with 10 \% FCS, 25 mM HEPES pH 6.9, 4 mM NaHCO $31 \mathrm{mM}$ glutamine, $1 \mathrm{x}$

340 RPMI 1640 vitamin mix, $0.2 \mu \mathrm{M}$ folic acid, $100 \mu \mathrm{M}$ adenine, $7.6 \mathrm{mM}$ hemin, $8 \mu \mathrm{M}$ biopterin, $34150 \mathrm{U} / \mathrm{ml}$ of penicillin, and $50 \mu \mathrm{g} / \mathrm{ml}$ of streptomycin. Promastigotes were then maintained in 342 culture by serial dilution in fresh medium once they reached stationary phase. At passages

3432,10 and 20 corresponding to approximately 20, 60 and 190 generations respectively,

344 parasites in exponential growth phase were collected and adjusted to $2 \times 10^{8}$ parasites per

345 tube for DNA extraction (see below). After 20 in vitro passages, serial dilutions of

346 promastigotes were plated on M199 Agar plates and 8 clones were selected and amplified in

347 promastigote medium.

349 Hamster infection and isolation of infectious amastigotes. We used female

350 RjHan:AURA golden syrian hamsters between 4 to 6 weeks of age (Janvier Labs, France)

351 and a weight between 60 to $90 \mathrm{~g}$. Housing of the animals and all experiments were 
conducted in agreement with the project number 2013-0092 approved by the Institut Pasteur

353 Ethics committee in accordance to the European legislation/guidelines EU 2010/63. We

354 used a total of 13 hamsters for DNA-FISH analysis (1), DNA/RNA extraction from purified

355 amastigotes (2), hamster infection (6), monitor tissue parasite burden (3), and comparative

356 HTseq analysis of liver and spleen amastigotes (1). Given the nature of our animal

357 experiments to compare infected versus non-infected animals and use infected animals as a

358 source of biological material, our studies were not blinded or randomized. No animals were

359 excluded from analysis. Anesthetized hamsters were inoculated by intra-cardiac injection

360 with $5 \times 10^{7}$ amastigotes obtained from infected hamster spleens. Hamster weight was

361 monitored and animals were euthanized with $\mathrm{CO}_{2}$ after four months of infection. Spleens and

362 livers were collected, weighed, and homogenized in PBS as described ${ }^{31}$. For amastigote

363 purification, tissues were homogenized in $25 \mathrm{ml}$ PBS supplemented with $2.5 \mathrm{mg} / \mathrm{ml}$ saponine

364 using the gentleMACS homogenizer and gentleMACS M tubes from Miltenyi. Homogenates

365 were cleared by centrifugation at $130 \mathrm{~g}$ for $5 \mathrm{~min}$ at $4^{\circ} \mathrm{C}$, the supernatants were collected, 1

$366 \mathrm{ml}$ of saponine $(25 \mathrm{mg} / \mathrm{ml})$ was added under gentle agitation, and parasites were harvested

$3675 \mathrm{~min}$ later by centrifugation at $2000 \mathrm{~g}$ for $10 \mathrm{~min}$ at $4^{\circ} \mathrm{C}$. After two washing steps with PBS,

368 remaining host cell contaminants were removed by Percoll centrifugation. Briefly, parasite

369 were resuspended in $6 \mathrm{ml}$ of $45 \%$ Percoll, and $3 \mathrm{ml}$ were layered on a cushions of $2 \mathrm{ml}$ of

$37090 \%$ Percoll in $15 \mathrm{ml}$ falcon tubes. After $35 \mathrm{~min}$ of centrifugation at $3500 \mathrm{~g}, 15^{\circ} \mathrm{C}$,

371 amastigotes were recovered from the interface of the gradient and washed 3 times in PBS

372 (centrifugation at $2000 \mathrm{~g}, 10 \mathrm{~min}, 4^{\circ} \mathrm{C}$ ). Tissue-derived amastigotes were adjusted to $2 \times 10^{8}$

373 parasites per tube for RNA or DNA extraction or inoculated in culture medium for

374 differentiation into promastigotes and further culture.

375 
376 DNA FISH analysis. DNA probes for chromosomes 5, 17, 22, and 27 were prepared as

377 described in $^{18}$. Amastigotes were purified from infected hamster livers and spleens

378 (previously inoculated with amastigotes obtained from infected hamster spleens) as

379 described above, immobilized on glass slides, fixed in $4 \%$ paraformaldehyde and $4 \%$ acetic

380 acid. Fluorescence in situ hybridization was performed according to Sterkers et al. ${ }^{18}$.

381 Leishmania cells were viewed by phase contrast, and fluorescence was visualized using

382 appropriate filters on a ZeissAxioplan 2 microscope with a 100 x objective. Digital images

383 were captured using a Photometrics CoolSnap CDD camera (Roper Scientific) and

384 processed with MetaView (Universal Imaging). Z-Stack image acquisitions (15 planes of

$3850.25 \mathrm{~mm}$ ) were systematically performed for each cell analyzed using a Piezo controller,

386 allowing to view the nucleus in all planes and to count the total number of labeled

387 chromosomes. The ploidy was estimated on 100 labeled cells. We carefully considered the

388 cell cycle state and excluded cells that were replicating their DNA as judged by the presence

389 of large or duplicated kinetoplasts, which corresponded to less than $1 \%$ and thus a negligible

390 fraction. This low replication rate was confirmed by our own infection data analysis that

391 allowed us to calculate a generation time of amastigotes in vivo of 10.4 days.

394 Genomic sequencing. All sequencing analysis were performed using Illumina short-read

395 technology. The sequences for BPK282/0 promastigotes and amastigotes shown in Figure

396 2a were generated using the Nextera XT library preparation kit (Illumina), the KAPA Library

397 Quantification Kits (KAPA Biosystems), and paired-end sequencing on a HTSeq 1500

398 sequencer (Illumina). The sequences of LD1S liver and spleen amastigotes (Figure 5c,

399 Supplementary Figure 8 and 9) were carried out as a service by the Centro Nacional de 
400 Análisis Genómico (CNAG, Barcelona) using the following protocol: 0.3-0.6 micrograms of 401 sheared genomic DNA (Covaris E201) was end-repaired, adenylated and ligated to Illumina 402 specific indexed paired-end adapters (adapter:insert molar ratio 40:1). The DNA library was 403 size selected with AMPure XP beads in order to reach an insert size of 220-550bp and to 404 remove unligated adapters. The final libraries were quantified with the Library Quantification 405 Kit (Kapa Biosystems). On the base of the qPCR quantification the molar concentration 406 sufficient for sequencing run was estimated. Each library was sequenced using TruSeq SBS 407 Kit v3-HS (Illumina Inc.), in paired end mode, 2x101bp, in a fraction of one sequencing lane 408 of an HTSeq2000 flowcell v3 (Illumina Inc.) according to standard Illumina operation 409 procedures with a yield between 3,593 - 6,326 Gb for each sample. The rest of the LD1S 410 Leishmania sequences were generated at the Centre for Genomic Regulation (CRG, 411 Barcelona) using the following protocol: DNA was isolated using DNeasy blood and tissue 412 kits from Qiagen, nucleic acid concentration was measured with a NanoDrop $®$ spectrometer, 413 and between 2 to $5 \mu \mathrm{g}$ were used for sequencing and quality control. DNA sequencing was 414 performed using an Illumina HiSeq 2000 platform and TruSeq v3 kits. One $\mu g$ of nucleic 415 acids was used for DNAseq while the rest of the material was used for quality control. All 416 libraries were sequenced in 125 bp reads. A summary of the read numbers and read-length 417 associated with each genome is provided in Supplementary Table 2.

418

419 Read depth Sequencing Analysis. All DNA sequences were mapped against $L$. donovani 420 BPK282A1 from TriTrypDBv7. Mapping and post processing was carried out using a 421 combination of BWA mem $(\mathrm{v} 0.7 .8)^{32}$ along with Samtools $(\mathrm{v} 1.3)^{33}$ in order to refine read 422 information and clean mapped sequences. Alignments were further refined using GATK 423 (v2.8) IndelRealigner and MarkDuplicates ${ }^{34}$. Samtools depth command was used to estimate 
424 read-depth for every base, so as to determine median read-depth for each chromosome. In

425 order to estimate chromosome polysomy level in each sample, chromosome 34 - which

426 showed a stable disomic level across samples - was used to normalize other chromosomes

427 read depth. This measure was calibrated to determine polysomy levels, under the

428 assumption that the most frequent polysomy levels correspond to disomy. Intermediate

429 polysomy levels were classified as trisomic or tetrasomic events by using cutoffs on the

430 normalized chromosome read depth values. Chromosomes with values ranging between 2.6

431 and 3.4 were classified as trisomic, and values between 3.6 and 4.4 as tetrasomic. These

432 calibrated values were used to select subsets of chromosomes having comparable

433 aneuploidy levels. (Accession number: PRJEA61817)

434

435 Aneuploidy co-occurrence. Aneuploidy co-occurrence analysis was carried out by

436 measuring the Pearson correlations between pairs of chromosomes, with each chromosome

437 being represented as vector of normalized depth values, each value being drawn from an

438 isolate. P-Values were measured so as to identify pairs having the highest levels of co-

439 variation - as opposed to constant or unrelated polysomy levels.

440

441 Variant calling and allele frequency analysis. Pileups were generated across the

442 reference genome for all the samples. Samtools mpileup and its multiallelic caller were then

443 used to detect variants separately in all samples. In order to filter out sites potentially

444 affected by episomal amplifications, regions with a read-depth higher than $1.6 \mathrm{x}$ the median

445 depth of each chromosome were excluded from the analysis. The resulting sites were then

446 used to draw allele frequency statistics, and produce allele frequency profiles. Variants were

447 then filtered using a quality threshold of 15 using a Read Position Bias (RPB) filter of 0.1 and 
448 alleles with frequencies comprised between 0.1 and 0.9 were retained. This approach, that

449 was explicitly designed to prioritize sensitivity over specificity, yields about 10 times more

450 sites than a more conservative approach ( 28071 vs 2418 sites $^{19,20}$ ). The expected rate of

451 false positive associated with this procedure can be shown to be in the order of $3 \%$ (-

$\left.45210^{*} \log 10(1 / 31.6)\right)$. This amount of noise that would be incompatible with biomarker discovery

453 does not compromise the current analysis focused on tracing homologue chromosome fate

454 in aneuploidies.

456 Merged allele frequency profiles. The allele frequencies associated with variants called as

457 explained in the previous section were then used to build merged allele frequency

458 distribution profiles for each chromosome across isolates. Profiles of homologue

459 chromosomes classified with the same polysomy level - as explained in read depth analysis

460 - were added up to yield a set of merged profiles (Supplementary Figure 1). The stacking of

461 pre-computed profiles presents the advantage of maintaining the individual segregation

462 profiles and can therefore be used to estimate profile homogeneity across samples as

463 indicated by well-defined peaks that reflect conserved allelic frequencies across isolates.

464 Most of these profiles exhibit the near canonical profiles expected for allele distribution in a

465 diploid, triploid or tetraploid organism and therefore confirm the suitability of the noise to

466 signal ratio of our site calling procedure.

467

468 Segregation variations and Loss of Heterozygosity dispersion plots. Segregation

469 variations (SV) between disomic and trisomic chromosomes were estimated by considering

470 for each heterozygous site the log ratio between the frequency of the most major (major

471 allele) allele in the considered isolate, and the average frequency of the same allele in the 
disomic population.

473

$474 \quad$ With

475

476

477

478

479

480

481

482

483

484

485

486

487

488

489

490

491

492

493

494

- $s$ heterozygote site in an isolate

- $t$ isolate trisomic for chromosome $\mathrm{N}$

- $T$ all the isolates trisomic for chromosome $\mathrm{N}$

- $d$ isolate disomic for chromosome $\mathrm{N}$

- D all the isolates disomic for chromosome $\mathrm{N}$

$-M A(s, t)$ the Major Allele on site $s$ in the isolate $t$ trisomic for Chromosome $N$

-Frequency $(M A(s, t), t)$ is the frequency of $M A(s, t)$ in isolate $t$

-AvgFrequency $(M A(s, t), D)$ the average frequency of $M A(s, t)$ across all isolates $D$ disomic for Chromosome $\mathrm{N}$

This procedure amounts to considering the disomic average as a null hypothesis with respect to the fate of the corresponding alleles in their trisomic states. When doing so the nature of the allele is not considered and two isolates having different major alleles on the same site can nonetheless contribute the same value to the distribution of ratios, as plotted in Supplementary Figure S1b. These ratios were then averaged for each chromosome and used as a measure for Segregation variation. On Supplementary Fig S1, profiles are ordered according to this ratio that tends to reflect the disomic tendency in the trisomics. 
495 trisomic isolates and the corresponding frequency across disomic isolates. These ratios

496 were then averaged for each chromosome and used as a loss of heterozygosity measure

497 using the following formula

498

499

500

501

502

503

504

505

506

507

508

509

510

511 This measure reflects the tendency of a given allele to dominate in frequency the overall

512 trisomic population. It makes it possible to discriminate between a scenario where the two

513 original disomic alleles are equally likely to have increased frequencies in each isolate (i.e.

514 random selection of the duplicated homologous chromosome), and situations where the

515 same allele is consistently amplified across all isolates (i.e. clonal amplification of the same

516 trisomy or consistent selection of the same homologous chromosome amplification). The

517 comparison between SV and LOH across field isolates is shown in Fig1d, with a p-value 
518 calculated as the t-test statistic based on Pearson's product moment correlation coefficient

519 using cor.test() function in R.

520

521 Haplotype phasing. Haplotype phasing was carried out by considering the effect of

522 aneuploidy on the original frequencies following the protocol originally defined in ${ }^{21}$. In this

523 analysis, alleles undergoing similar shifts are considered as being part of the same

524 haplotype block. While heterozygous SNPs are not dense enough in the 204 filed isolates for

525 this approach to be validated, we took advantage of the LD1S hybrid origin to verify that

526 SNPs occurring with the insert distances of the pair-ended sequencing had similar

527 segregation shifts after duplication and could therefore considered to be genetically linked,

528 thus ruling out recombination as a major confounding factor. This finding is in agreement

529 with the original study of very low recombination rates in the 204 filed isolates. Our analysis

530 indicates that $50 \%$ of the SNP pairs within insert distance of one another have allelic

531 frequencies less that 10\% point apart in the bimodal trisomics (Fig S11), as compared with

$53223 \%$ when randomizing pair ends in order to test for pair connectivity and allele linkage.

533

534 Transcriptome sequencing. RNA was prepared using RNeasy mini plus blood and tissue

535 kits from Qiagen according to manufacturer instructions. Nucleic acid concentrations were

536 measured with a NanoDrop $®$ spectrometer. RNA sequencing was performed using an

537 Illumina Hiseq 2000 platform and TruSeq v3 kits. Four $\mu g$ of nucleic acids were used for

538 RNAseq and the rest of the material was used for the quality control. RNA libraries were

539 sequenced on a single flow cell single stranded $51 \mathrm{bp}$ read. Sequencing reads have been

540 submitted to the European Nucleotide Archive (ENA) and are available under the submission

541 number PRJEB15282. The amount and the length of reads obtained from each analysis are 
542 summarized in the Supplementary Table 2.

543

544 Relative expression estimation. Gene copy numbers and expression levels were

545 estimated from DNA and RNA read counts respectively, mapping to annotated ORFs using

546 reference gene annotation from TriTrypDBv7. The actual read-counts were measured with

547 BedTools (v2.19) ${ }^{35}$ using the BAM files obtained after mapping. In order to normalize for

548 gene length, read counts were estimated in Reads Per Kilobase Mapped (RPKM) ${ }^{36}$ across

549 all genes and samples. These RPKM measurements were then used to compare expression

550 levels across samples.

551

552 Data availability. All Sequencing reads have been submitted to the European Nucleotide

553 Archive (ENA) and are available under the Accession Number PRJEB15282. The amount

554 and the length of reads obtained from each analysis are summarized in the Supplementary

555 Table 2.

556

557 Code availability

558 Custom scripts and code necessary to generate figures and files can be found in the

559 following repository: https://github.com/pprieto/genomevoleish

560

561 Biography

562 1. Pallen, M. J. \& Wren, B. W. Bacterial pathogenomics. Nature 449, 835-842 (2007).

563 2. Torres, E. M. et al. Effects of aneuploidy on cellular physiology and cell division in

564 haploid yeast. Science 317, 916-924 (2007).

565 3. Pavelka, N. et al. Aneuploidy confers quantitative proteome changes and phenotypic 
variation in budding yeast. Nature 468, 321-325 (2010).

567 4. Pavelka, N., Rancati, G. \& Li, R. Dr Jekyll and Mr Hyde: role of aneuploidy in cellular $568 \quad$ adaptation and cancer. Curr. Opin. Cell Biol. 22, 809-815 (2010).

569 5. Selmecki, A. M., Dulmage, K., Cowen, L. E., Anderson, J. B. \& Berman, J. Acquisition of 570 aneuploidy provides increased fitness during the evolution of antifungal drug resistance.

$571 \quad$ PLoS Genet. 5, e1000705 (2009).

572 6. Sabeti, P. C. et al. Detecting recent positive selection in the human genome from 573 haplotype structure. Nature 419, 832-837 (2002).

574 7. Staaf, J. et al. Landscape of somatic allelic imbalances and copy number alterations in 575 human lung carcinoma. Int. J. Cancer 132, 2020-2031 (2013).

576 8. Scheinfeldt, L. B. \& Tishkoff, S. A. Recent human adaptation: genomic approaches, 577 interpretation and insights. Nat. Rev. Genet. 14, 692-702 (2013).

578 9. Gao, R. et al. Punctuated copy number evolution and clonal stasis in triple-negative $579 \quad$ breast cancer. Nat. Genet. 48, 1119-1130 (2016).

580 10. Sterkers, Y., Crobu, L., Lachaud, L., Pagès, M. \& Bastien, P. Parasexuality and mosaic 581 aneuploidy in Leishmania: alternative genetics. Trends Parasitol. 30, 429-435 (2014).

582 11. Imamura, H. et al. Evolutionary genomics of epidemic visceral leishmaniasis in the $583 \quad$ Indian subcontinent. Elife 5, (2016).

584 12. Alvar, J. et al. Leishmaniasis worldwide and global estimates of its incidence. PLoS One $585 \quad 7$, e35671 (2012).

586 13. Zilberstein, D. \& Shapira, M. The role of $\mathrm{pH}$ and temperature in the development of 587 Leishmania parasites. Annu. Rev. Microbiol. 48, 449-470 (1994).

588 14. Leprohon, P., Fernandez-Prada, C., Gazanion, É., Monte-Neto, R. \& Ouellette, M. Drug 589 resistance analysis by next generation sequencing in Leishmania. Int. J. Parasitol. 
Drugs Drug Resist. 5, 26-35 (2015).

591 15. Downing, T. et al. Whole genome sequencing of multiple Leishmania donovani clinical 592 isolates provides insights into population structure and mechanisms of drug resistance. Genome Res. 21, 2143-2156 (2011).

594

16. Dujardin, J.-C., Mannaert, A., Durrant, C. \& Cotton, J. A. Mosaic aneuploidy in Leishmania: the perspective of whole genome sequencing. Trends Parasitol. 30, 554555 (2014).

17. Rogers, M. B. et al. Chromosome and gene copy number variation allow major structural change between species and strains of Leishmania. Genome Res. 21, 2129$2142(2011)$.

18. Sterkers, Y., Lachaud, L., Crobu, L., Bastien, P. \& Pagès, M. FISH analysis reveals aneuploidy and continual generation of chromosomal mosaicism in Leishmania major. Cell. Microbiol. 13, 274-283 (2011).

19. Imamura, H. et al. Evolutionary genomics of epidemic visceral leishmaniasis in the $604 \quad$ Indian subcontinent. Elife 5, (2016).

605 20. Dumetz, F. et al. Modulation of Aneuploidy in Leishmania donovani during Adaptation to 606 Different In Vitro and In Vivo Environments and Its Impact on Gene Expression. MBio 8, $607 \quad$ (2017).

608 21. Legrand, M. et al. Haplotype mapping of a diploid non-meiotic organism using existing 609 and induced aneuploidies. PLoS Genet. 4, e1 (2008).

610 22. Yona, A. H. et al. Chromosomal duplication is a transient evolutionary solution to stress. $611 \quad$ Proc. Natl. Acad. Sci. U. S. A. 109, 21010-21015 (2012).

612 23. Sterkers, Y. et al. Novel insights into genome plasticity in Eukaryotes: mosaic 613 aneuploidy in Leishmania. Mol. Microbiol. 86, 15-23 (2012). 
614 24. Rougeron, V., De Meeûs, T., Kako Ouraga, S., Hide, M. \& Bañuls, A.-L. ‘Everything you 615 always wanted to know about sex (but were afraid to ask)' in Leishmania after two 616 decades of laboratory and field analyses. PLoS Pathog. 6, e1001004 (2010).

617 25. Lande, R. Natural Selection and Random Genetic Drift in Phenotypic Evolution. $618 \quad$ Evolution 30, 314-334 (1976).

619 26. Lynch, M. \& Conery, J. S. The evolutionary fate and consequences of duplicate genes. $620 \quad$ Science 290, 1151-1155 (2000).

621 27. Victoir, K. \& Dujardin, J.-C. How to succeed in parasitic life without sex? Asking 622 Leishmania. Trends Parasitol. 18, 81-85 (2002).

623 28. Bastien, P., Blaineau, C. \& Pages, M. Leishmania: sex, lies and karyotype. Parasitol. $624 \quad$ Today 8, 174-177 (1992).

625 29. Dujardin, J.-C. et al. Clonal propagation and the fast generation of karyotype diversity: 626 An in vitro Leishmania model. Parasitology 134, 33-39 (2007).

627 30. Gazanion, É., Fernández-Prada, C., Papadopoulou, B., Leprohon, P. \& Ouellette, M. 628 Cos-Seq for high-throughput identification of drug target and resistance mechanisms in 629 the protozoan parasite Leishmania. Proc. Natl. Acad. Sci. U. S. A. 113, E3012-21 $630 \quad$ (2016).

631 31. Pescher, P., Blisnick, T., Bastin, P. \& Späth, G. F. Quantitative proteome profiling 632 informs on phenotypic traits that adapt Leishmania donovani for axenic and intracellular 633 proliferation. Cell. Microbiol. 13, 978-991 (2011).

634 32. Li, H. \& Durbin, R. Fast and accurate short read alignment with Burrows-Wheeler 635 transform. Bioinformatics 25, 1754-1760 (2009).

636 33. Li, H. et al. The Sequence Alignment/Map format and SAMtools. Bioinformatics 25, $637 \quad 2078-2079$ (2009). 
638 34. McKenna, A. et al. The Genome Analysis Toolkit: a MapReduce framework for

639 analyzing next-generation DNA sequencing data. Genome Res. 20, 1297-1303 (2010).

640 35. Quinlan, A. R. \& Hall, I. M. BEDTools: a flexible suite of utilities for comparing genomic

641 features. Bioinformatics 26, 841-842 (2010).

642 36. Mortazavi, A., Williams, B. A., McCue, K., Schaeffer, L. \& Wold, B. Mapping and

643 quantifying mammalian transcriptomes by RNA-Seq. Nat. Methods 5, 621-628 (2008).

644 


\section{Authors Contribution}

646 P.P.B., P.P, C.N. and G.F.S worked on all aspects of work, contributed to the design of the 647 project and wrote the article, G.B contributed to in silico analysis, F.D. performed hamster 648 infection experiment with field isolate, H.I. and J.C.D. contributed to the field isolates 649 analyses and revised the paper, D.K. helped analyzing the sequencing data, H.H. was 650 responsible for the genomic sequencing of the in vitro clones, V.C, P.B. and Y.S. contributed 651 the DNA-FISH analysis. Work on samples from the Indian sub-continent was supported by 652 the EU FP7 (Kaladrug-R, contract 222895), the Belgian Science Policy Office (TRIT, P7/41), 653 the Department of Economy, Science and Innovation in Flanders (ITM-SOFIB) and the

654 Flemish Fund for Scientific Research (G.0.B81.12). We thank Life Science Editors for editing 655 assistance.

656

\section{Funding}

658 Plan Nacional [BFU2011-28575 to C.N., P.P.B., D.K.]; Center for Genomic Regulation 659 (CRG); Spanish Ministry of Economy and Competitiveness, 'Centro de Excelencia Severo 660 Ochoa 2013-2017' [SEV-2012-0208]; Center for Genomic Regulation (CRG) (C.N, P.P.B.,

661 D.K). Grant from the Institut Pasteur International Department strategic fund to the LeiSHield 662 consortium (P.P.B, P.P, C.N., G.F.S., G.B., Y.S.). Agence Nationale de la Recherche (ANR) 663 within the frame of the "Investissements d'avenir" program (ANR-11-LABX-0024-01

664 "PARAFRAP") (V.C.,P.B., Y.S.). The EU FP7 (Kaladrug-R, contract 222895), the Belgian 665 Science Policy Office (TRIT, P7/41), the Department of Economy, Science and Innovation in 666 Flanders (ITM-SOFIB) and the Flemish Fund for Scientific Research (G.0.B81.12) (J.C.D., 667 F.D., H.I., M.D.).

668 
669 Competing interests

670 The authors declare no competing financial interests.

671

$672 \quad$ Figures

673 Figure 1: Genome instability, aneuploidy co-occurrence and haplotype selection in 204 L.

674 donovani field isolates. Polysomy analysis based on read-depth comparison and derived

675 allele frequency analysis on specific aneuploidies is shown. (a) Polysomy analysis.

676 Polysomy level was estimated for each chromosome and sample by read-depth analysis.

677 The distribution of chromosome copy number is shown across all field isolates. (b) Co-

678 occurrence analysis. Pearson correlation measured for each pair of chromosomes was

679 plotted while considering the median read depth measured in every field isolate. Empty

680 positions correspond to either uncorrelated pairs or insignificant correlations (significance

681 level of 0.05$)$. One main cluster appears containing typically trisomic chromosomes. (c)

682 Allele frequency profiles. Merged allele frequency distributions are shown for chromosomes

6831 and 5 for both disomic (right) and trisomic (left) states. (d) Segregation variation vs allele

684 diversity analysis. This panel summarizes the relationship between segregation variations

685 (SV, reflecting bi-modality of the merged frequency profiles) and loss of heterozygosity

686 ( $\mathrm{LOH}$, reflecting allelic diversity) when comparing trisomic and disomic isolates across 204

687 field isolates and the 36 chromosomes of the genome. For most chromosomes, both

688 quantities are highly correlated as indicated by the blue regression line.

689

690 Figure 2: In vivo aneuploidy dynamics. The three panels document the dynamic nature and 691 the mosaicism of $L$. donovani aneuploidy in situ during hamster infection. (a) Aneuploidy

692 pattern of $L$. donovani field isolate BPK282/0 after over 20 passages in culture (in vitro, iv) 
693 and following three consecutive passages of these $\mathrm{p}>20$ parasites in the hamster $(\mathrm{H} 3)$. Heat

694 maps show median normalized read depths of the 36 chromosomes in both samples and an

695 estimation of the polysomy level of each chromosome: blue, two copies; green, three copies;

696 yellow four copies. (b) DNA-FISH analysis. L. donovani strain LD1S amastigotes purified

697 from infected hamster liver (L) and spleen (S) were analyzed with fluorescent labeled probes

698 specific for chromosomes (chr) 5, 17, 22, and 27 and signals were analyzed by microscopy.

699 The signal for chr 5 in spleen-derived amastigotes is shown as an example (phase, phase

700 contrast; FISH, fluorescent signal from DNA-FISH analysis; overlay, merged image of DNA-

701 FISH and nuclear signal obtained with DAPI stain). The bar corresponds to $2 \mu \mathrm{m}$. The \%

702 chromosome number was calculated counting 100 individual cells per condition (lower

703 panel). The polysomy level is indicated by the bar filling, with white for monosomy, gray for

704 disomy and black for trisomy.

705

706 Figure 3. In vitro aneuploidy dynamics. This figure shows read-depth variations in $L$.

707 donovani LD1S genes (8760 genes) across chromosomes at different passages during

708 culture adaptation (4 samples) and in the 8 sub-clones derived from the parasite population

709 at passage 20. Chromosome gene read-depth distributions are shown in boxplots depicting

710 upper and lower quartiles and the median. (a) Read depth variation during culture

711 adaptation. Variations in read-depth for L. donovani LD1S amastigotes isolated from infected

712 hamster spleen (sp-ama) and derived promastigotes at passage 2, 10, and 20 (p2, p10,

713 p20). Only aneuploid chromosomes are shown (see full panel in Supplementary Fig. 3a).

714 Normalized read depth for every gene within each chromosome is displayed using a

715 standard box-plot representation with the central line representing the median of the

716 distribution. (b) Read depth variation on individual sub-clones. Variations on these same 
717 chromosomes as shown in panel A are shown for 8 individual parasite cultures subcloned

718 from p20 parasites (sub-clones are indicated by CL, for full panel see Supplementary Fig.

$7193 b)$.

720

721 Figure 4: Fluctuations of allele frequency during culture adaptation. These panels document

722 haplotype selection by following the allele frequency distribution in 8 sub-clones and the

723 original p20 parasite population ( $\mathrm{n}=9$ samples in total) across . (a) Haplotype selection in

724 clones. Variable sites in chromosomes undergoing amplification were either colored

725 according to the major alleles (i.e. highest frequency) or left in gray for balanced

726 heterozygote sites. Each painted pattern represents a chromosome, either from the original

727 p20 population (first line) or one of the 8 derived sub-clones. Allele frequency distributions

728 for each sample are shown on the left of each panel. The color code of the distributions

729 corresponds to the read depth, with disomic-to-trisomic-to-tetrasomic transitions indicated by

730 a continuum colored from red to green. The number of heterozygous sites for each

731 chromosome and sample are shown by the histograms (right side of each panel). Samples

732 with low counts as observed for chromosomes 15 and 20 represent loss of heterozygosity

733 occurring during culture adaptation. (b) Haplotype selection during culture adaptation. Allele

734 frequency distributions corresponding to hamster-derived splenic amastigotes (sp-ama) and

735 derived promastigotes during culture adaptation at passage p2, p10 and p20 are shown for

736 selected chromosomes. All chromosomes undergoing polysomy variations are displayed in

737 Supplementary Fig. 3.

738

739 Figure 5: Aneuploidies and haplotype selection correlate with phenotype and fitness. This

740 figure shows the relationship between aneuploidy and haplotype selection with 
741 transcriptomic output, in vitro and in vivo growth, and tissue-specific parasite adaptation. (a)

742 Correlation of aneuploidy and transcript output. Each dot corresponds to a chromosome with

743 the $\mathrm{X}$-axis reflecting the transcriptome median read-depth and the $\mathrm{Y}$-axis the corresponding

744 genomic median read-depth, as estimated for the eight subclones. A subset of

745 chromosomes was colored as indicated by the legend. (b) Parasite growth phenotype in vitro

746 and in vivo. LD1S promastigotes during culture adaptation at passages p2, p10, and p20

747 were assessed for in vitro growth to determine the generation time (left panel). Three

748 independent triplicate experiments were performed and results of one representative

749 experiment with standard deviation represented by the error bars is shown. In vivo parasite

750 growth was assessed in infected hamster spleens and livers by limiting dilution assay

751 (middle panel). The plot represents the mean parasite burden +/- SD of three infected

752 hamsters. Pathogenicity was monitored following hamster weight as a function of time (right

753 panel) for three non-infected and three infected hamsters. (c) Tissue-specific haplotype

754 selection. Amastigotes were isolated from liver and spleen of one infected hamster, purified

755 genomic DNA was subjected to HTseq analysis, and allele profiles were established by

756 plotting allele density versus frequency.

757

758

759 Figure 6: Aneuploidy influence on heterozygosity. Each chromosome is represented by its

760 number on the graph and colored according to aneuploidy frequency after culture

761 adaptation, as measured in the 204 L. donovani field isolates. The two lines (blue and green)

762 show apparent regimens of mutation rates. The green line fits a linear model for

763 chromosomes with an aneuploidy frequency higher than $25 \%$, while the blue line indicates

764 chromosomes that are lower in frequency. Chromosome 31 is excluded from the fitted 
765 models as it stands out as the only chromosome with aneuploidy in all samples.

766 


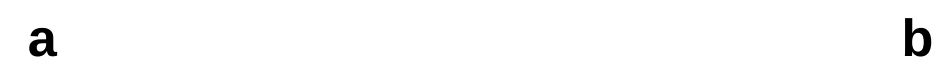$$
\text { . }
$$

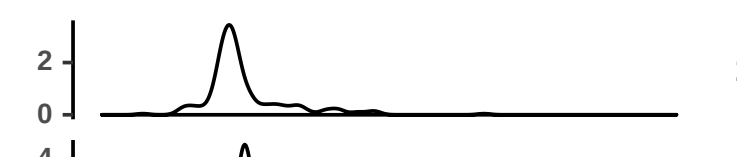

2
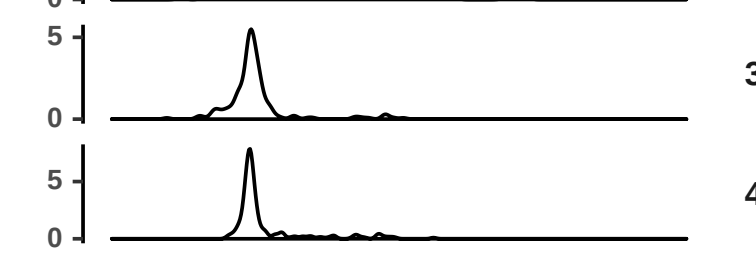

$\left.\begin{array}{l}1.0 \\ 0.5 \\ 0.0\end{array}\right]$
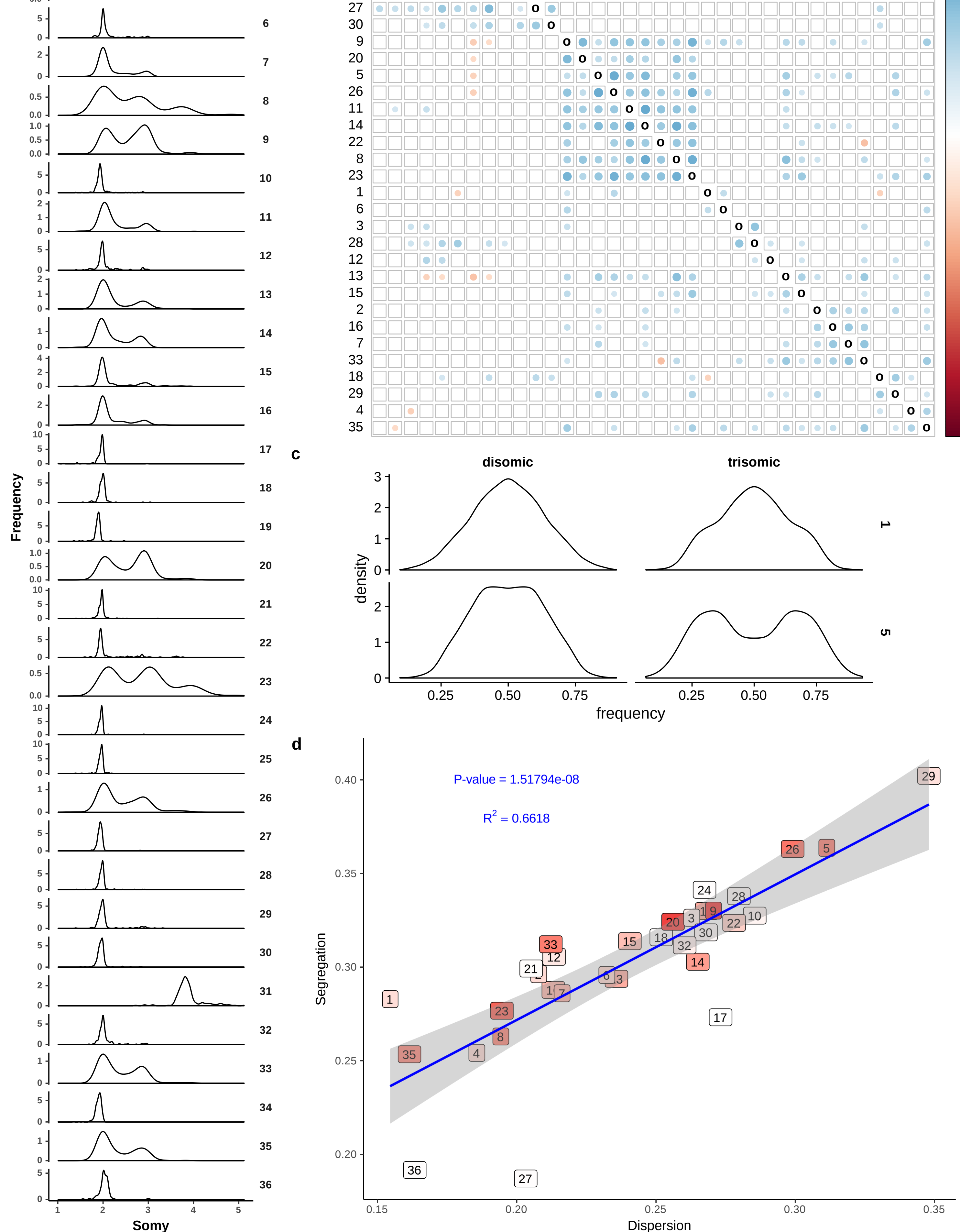

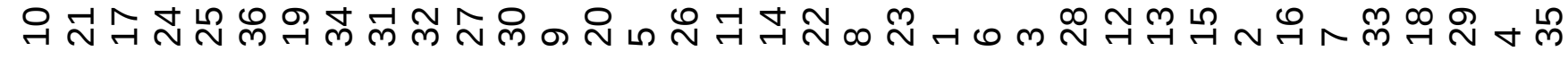

10 o

$210 \mathbf{0}$ 0

17 ○

24
25

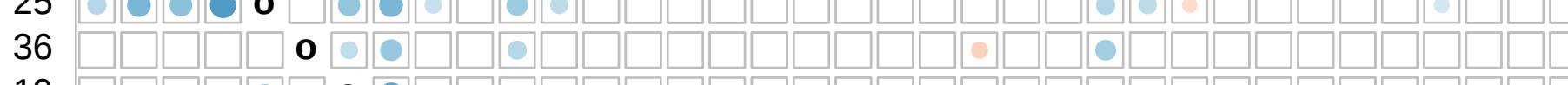

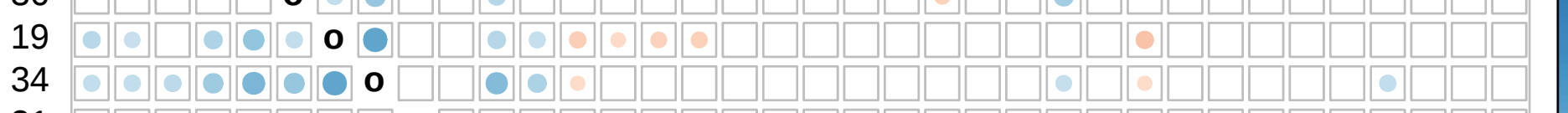

34
31 $\mathbf{0}$ 0

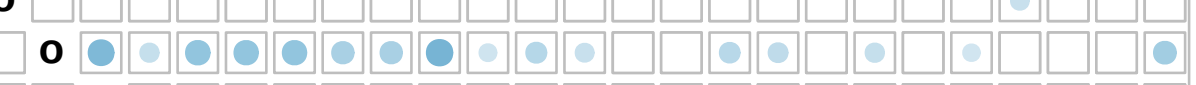

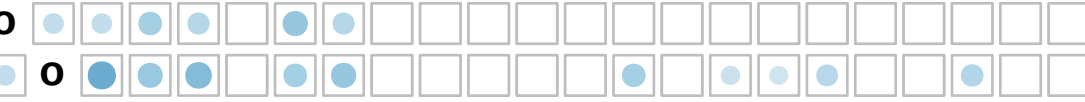

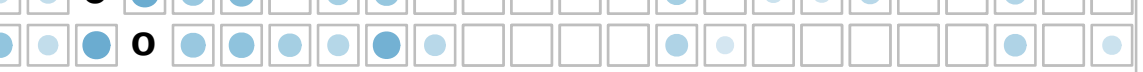

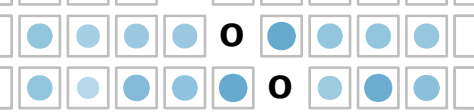

$0 \square \square 00000$

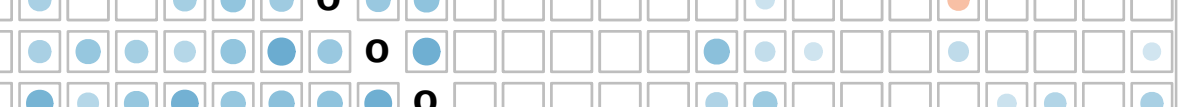
$\mathbf{0} 0 \square \square \square \square \square \square \square \square \square \square \square \square \square$

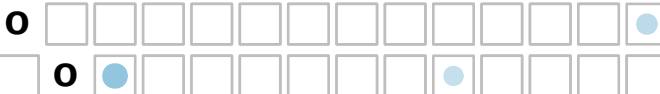

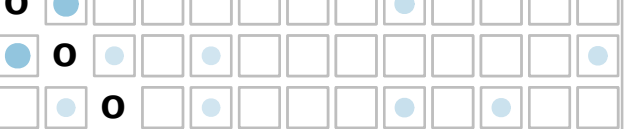
$0000 \square 0 \square$

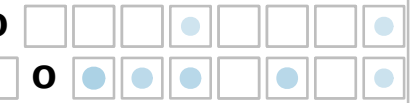
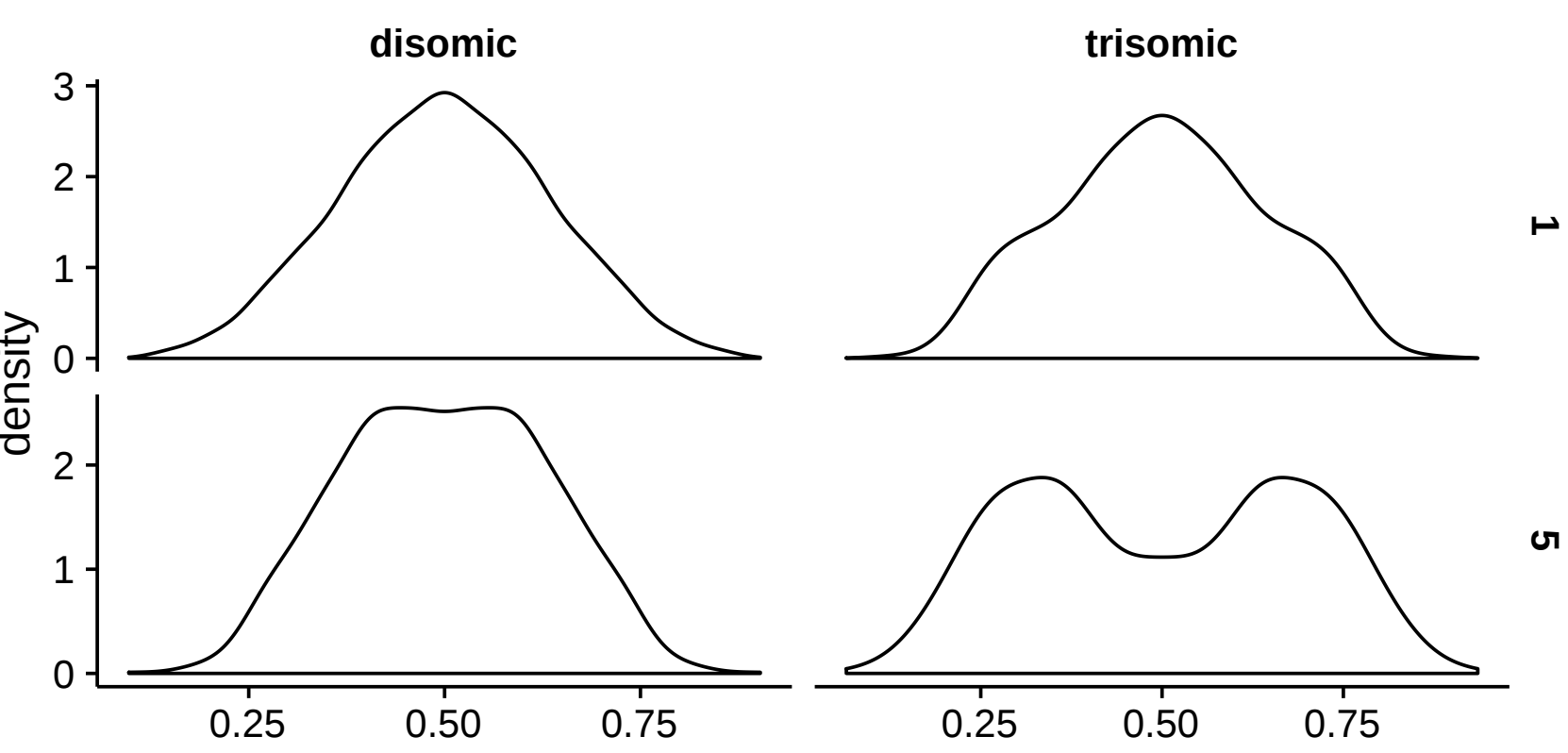

20

21

22

23

24

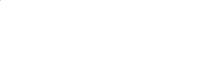




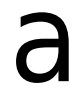

Polysomy level

$\begin{array}{lll}2 & 3 & 4\end{array}$

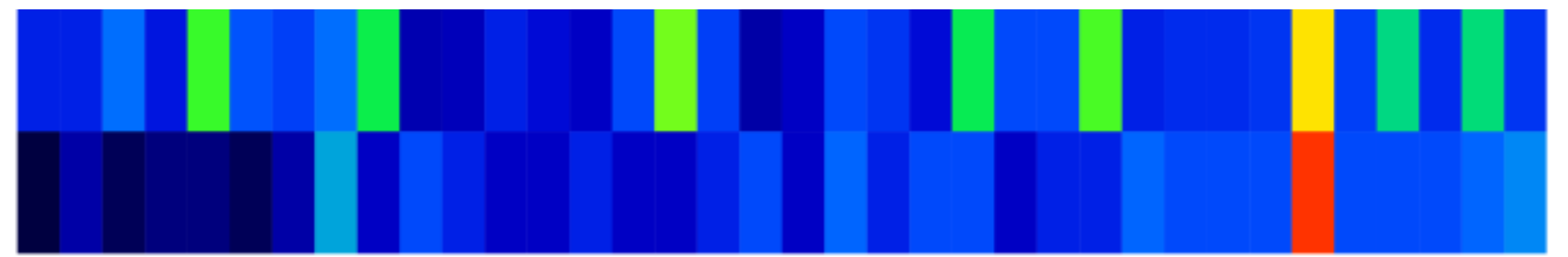

$122 \quad 3 \quad 4 \quad 5 \quad 6 \quad 7 \quad 8 \quad 9101112131415161718192021222324252627282930313233343536$ Chromosome
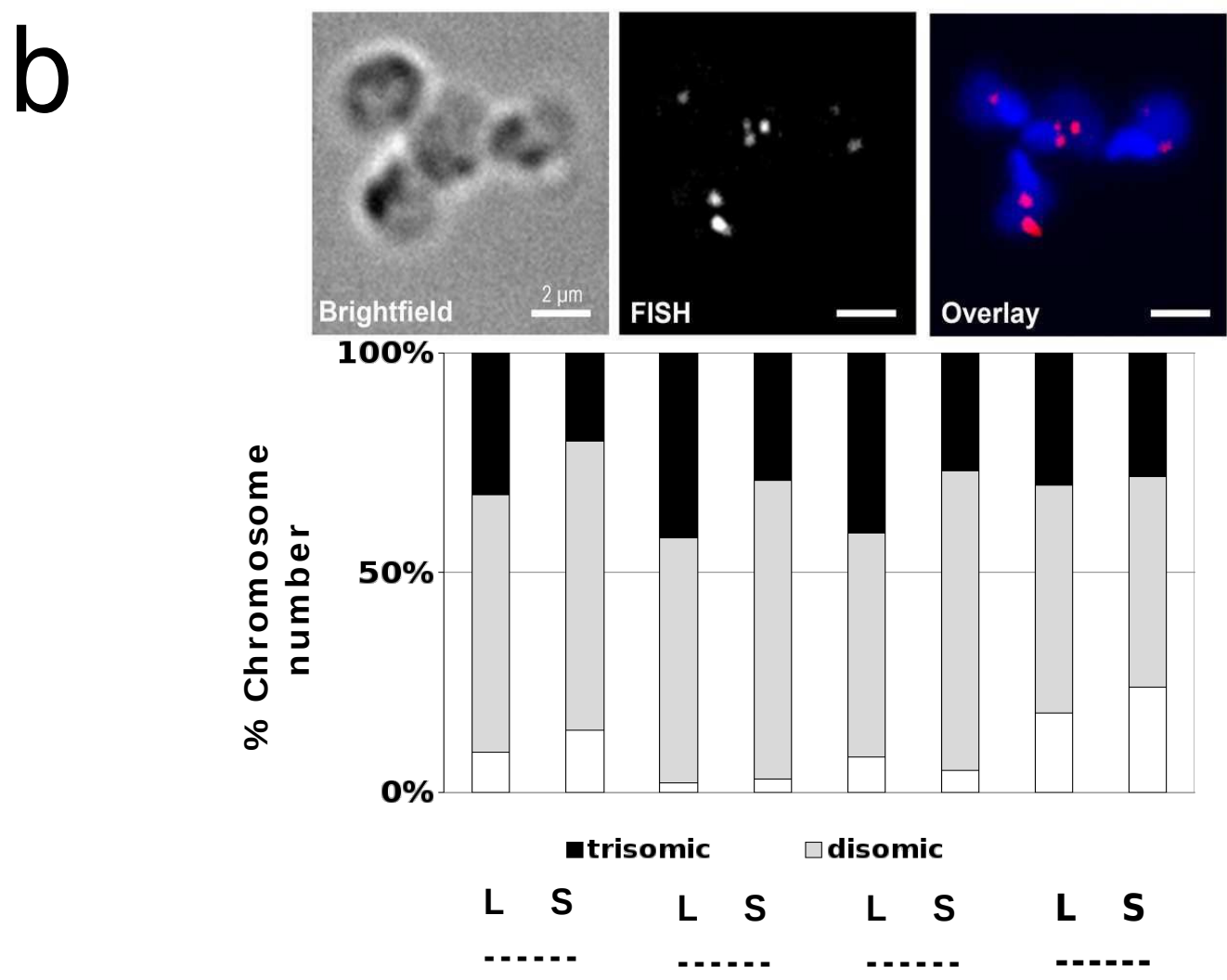

Chr 5

Chr 17

Chr 22

Chr 27 
9
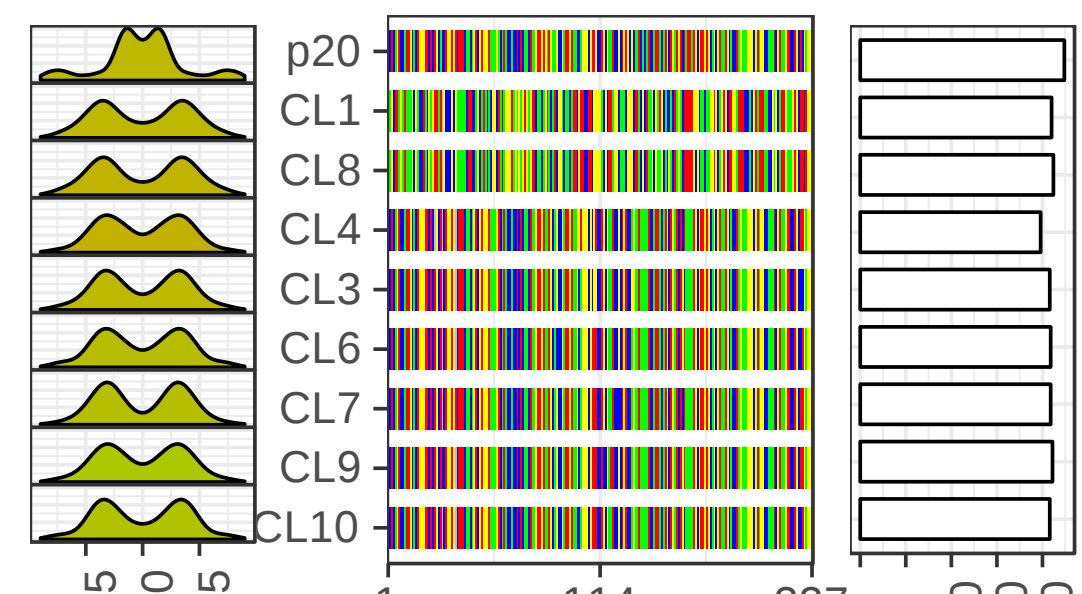

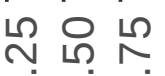
000

114 227 윰억윰유
1

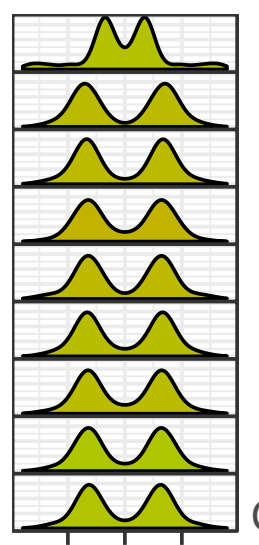

뉸ำ

$\circ 0^{\circ}$ p20-

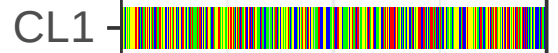

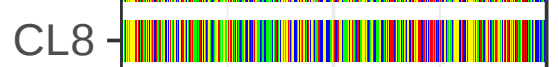

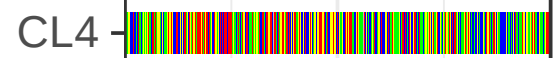
CL3 CL6 - -|||||||||||||||||||||||||||||||||||||||||||||||||||||||| $\mid$

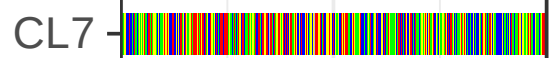

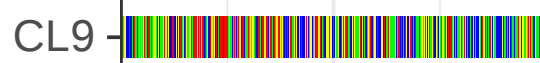

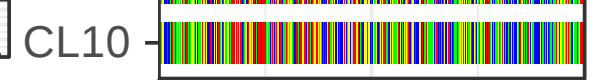

15

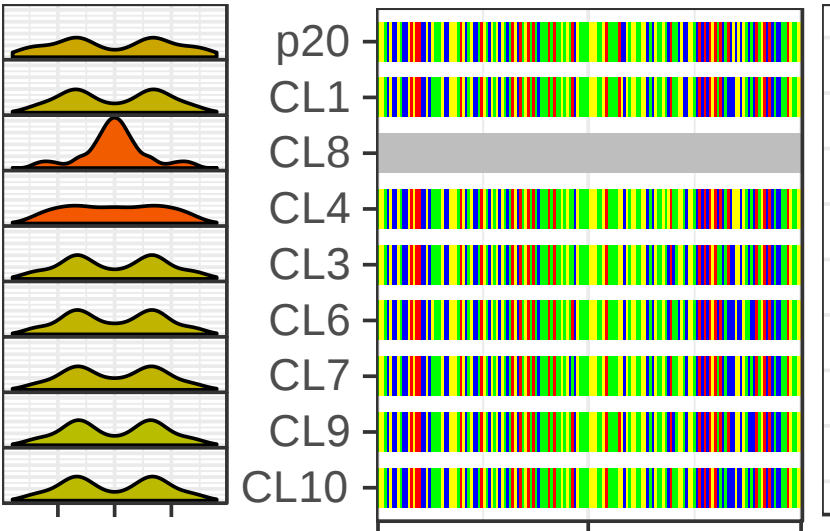

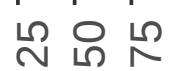

000

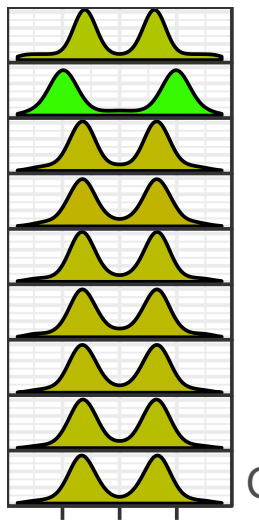

p20 -

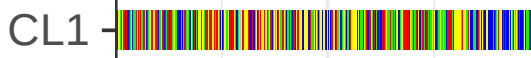

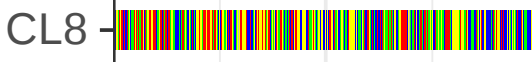

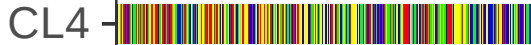

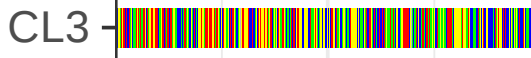

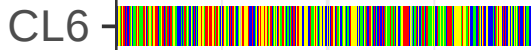

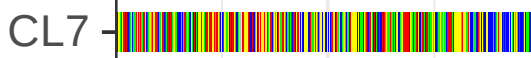

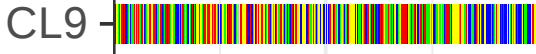
뉴용 농 CL10 -

$\circ \circ 0^{\circ}$

\begin{abstract}
252
\end{abstract}

\begin{abstract}
505
\end{abstract}

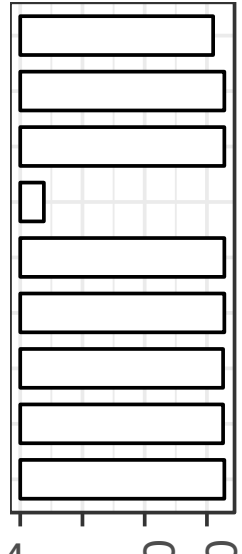

$164 \circ$ 융요육
20

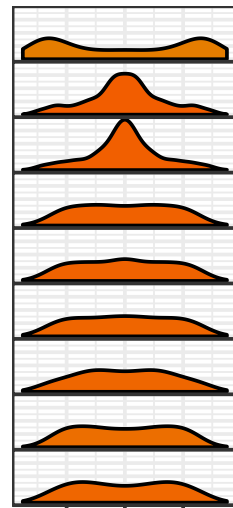

는ํำ

$\circ 0^{\circ}$ CL1 -
313
31

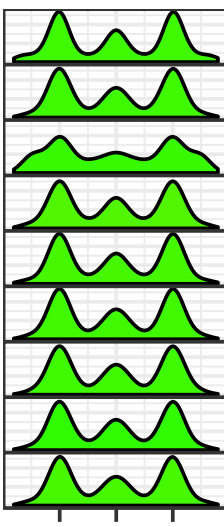

늉ํำ 눙

000 p20 CL10 -

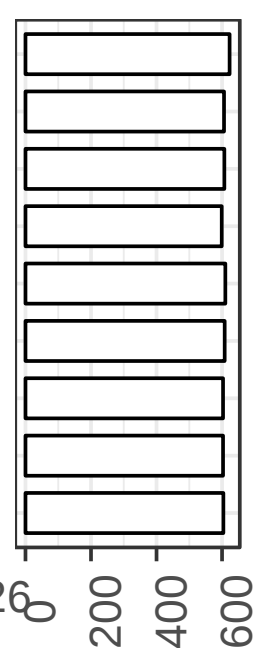

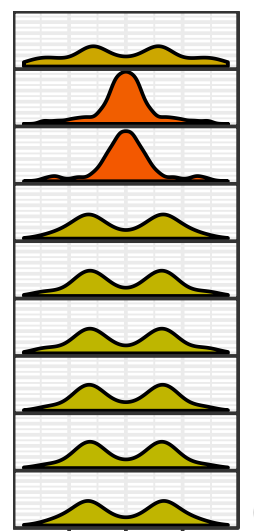

농용ํㅇ

000 p20 CL1

CL4 -||||||||||||||||||||||||||||||||||||||||||||||||||||||||||||||| CL3 - |||||||||||||||||||||||||||||||||||||||||||||||||||||||||||||||

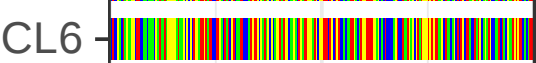
CL7-

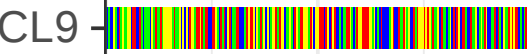

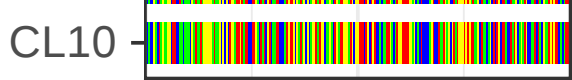

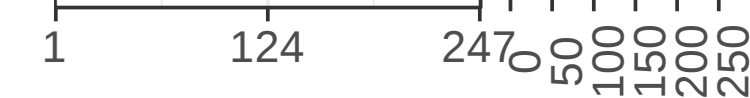

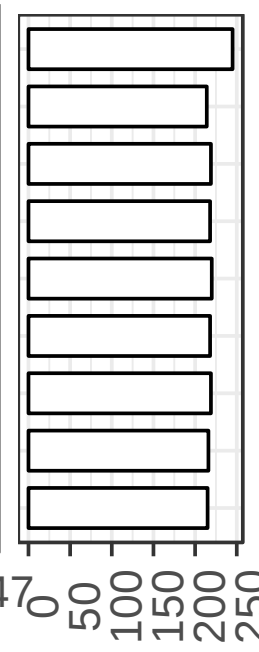

23

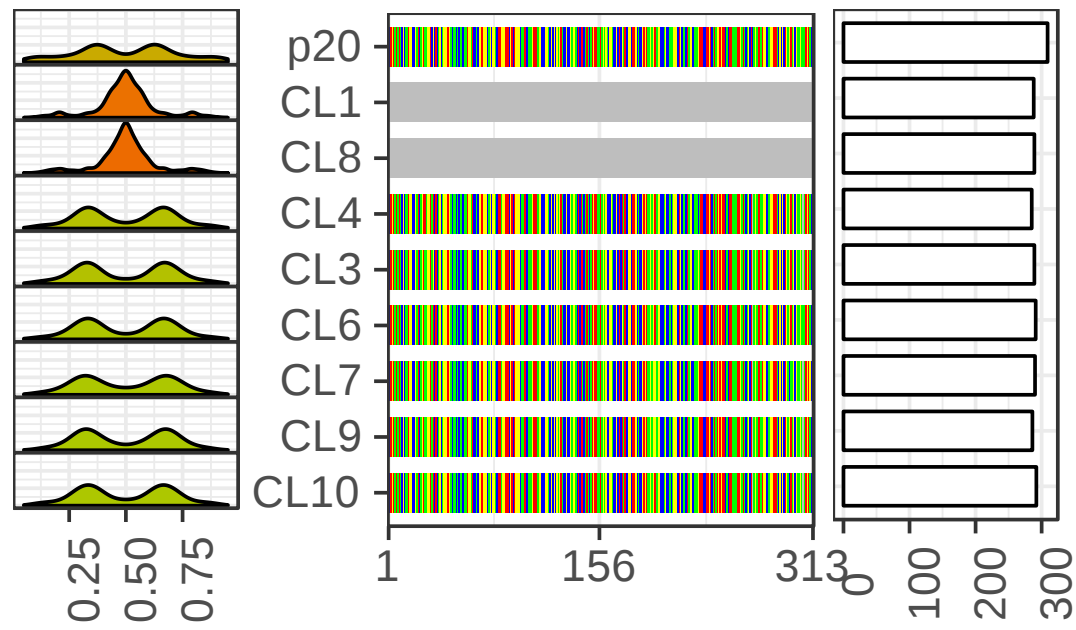

\section{| 20 -||||||||||||||||||||||| || || |||}

CL4 - || || ||||||||||||||||||| | | | | |||| || CL3 - III || || ||||||||||||||||| | | | |||| |

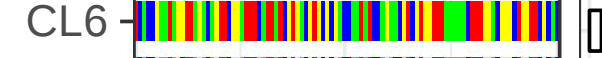
CL7 - ||||| || || ||||||||||||||| | | || ||| ||

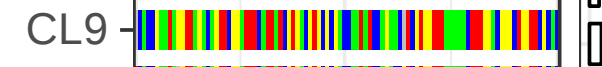
CL10 - IIIIIII ||||||||||||||||| || III|| |]

$50 \quad 100$ 융ㅇㅇㅇㅛ

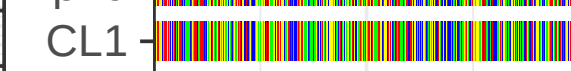

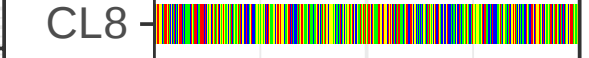

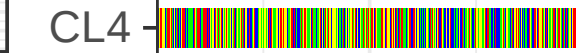
CL3 CL6 CL7 CL9 -

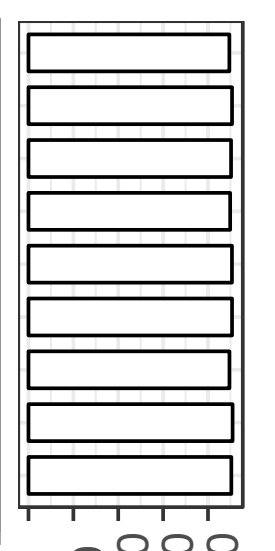

$11220 \quad 244$ 융윰용

b

Sample $\square$ splenic amastigote $\quad \square$ p2 $\square$ p10 $\square$ p20
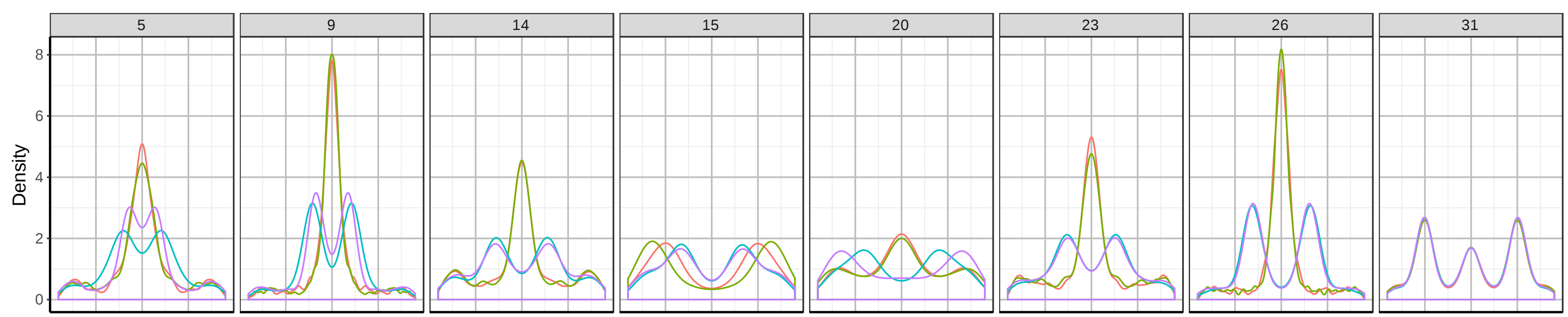

Frequency 


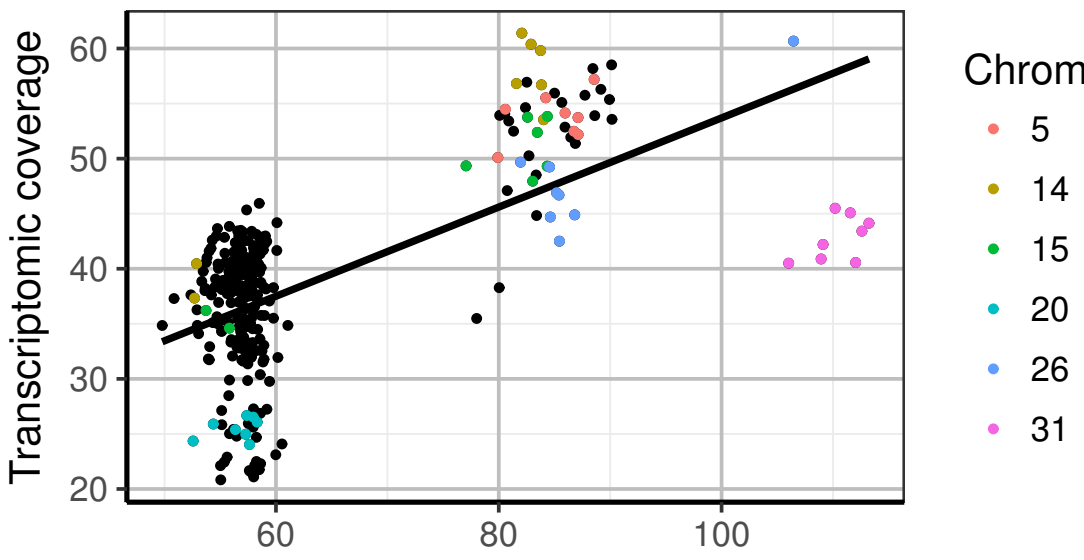

Genomic coverage
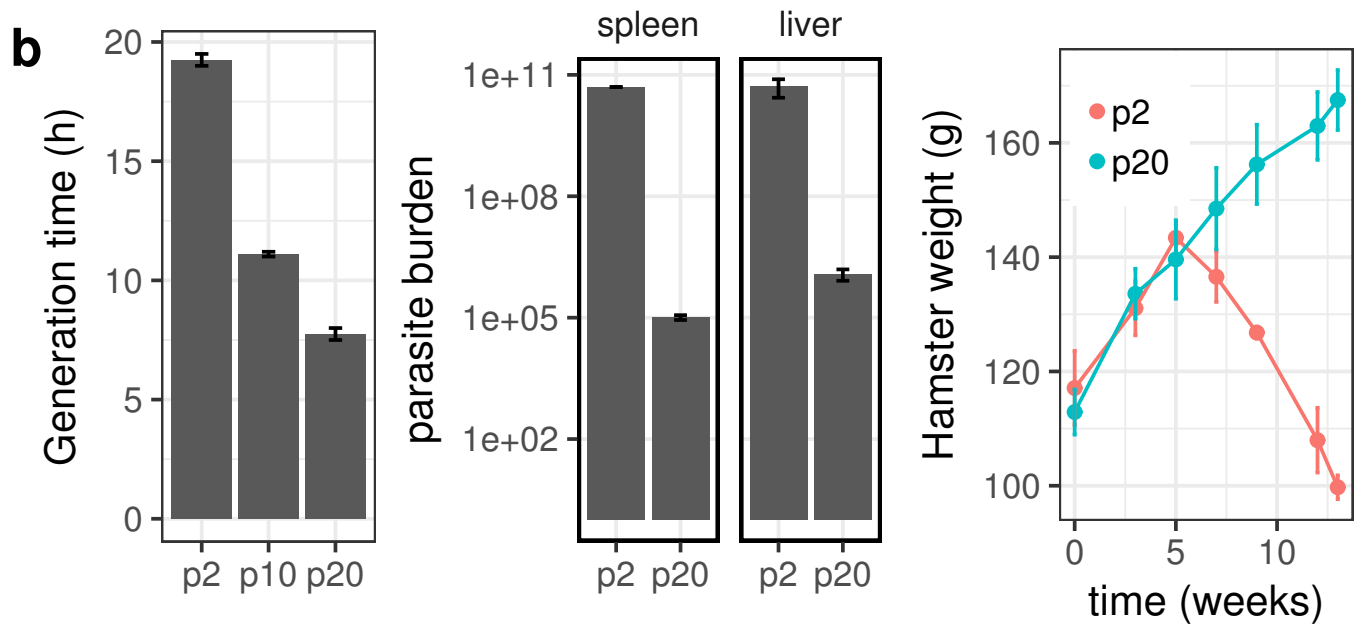

C
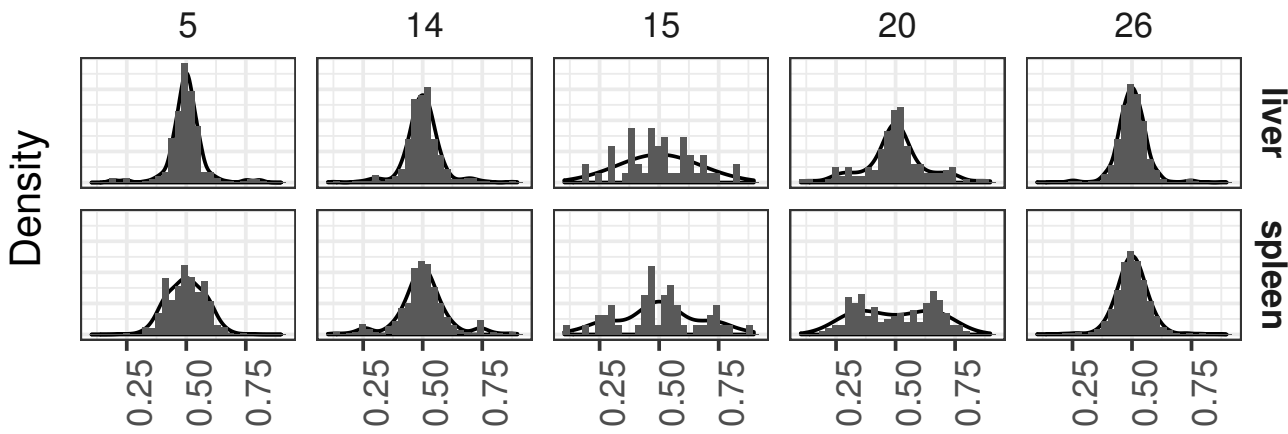

Frequency
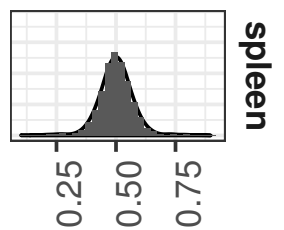
